\title{
Multicell Cooperation Based SVD Assisted Multi-User MIMO Transmission
}

\author{
W. Liu, S. X. Ng and L. Hanzo \\ School of ECS, University of Southampton, SO17 1BJ, United Kingdom. \\ Tel: +44-23-8059 6671, Fax: +44-23-8059 4508 \\ Email: \{wl03r,sxn,lh\}@ecs.soton.ac.uk, http://www-mobile.ecs.soton.ac.uk
}

\begin{abstract}
In this treatise, we investigated the application of singular value decomposion (SVD) assisted multiuser transmission in a multicell scenario. The SVD based scheme is capable of completely removing the cochannel interference, similarly to the classic zero forcing (ZF) based and block diagonalization (BD) aided schemes. Two different power allocation schemes are investigated for both SVD, ZF and BD based multicell transmssion. The SVD scheme achieves a suboptimal performance, but at a reduced complexity. Nonetheless, it always outperforms the $\mathrm{ZF}$ based scheme due to the joint reception of the transmitted symbols.
\end{abstract}

\section{INTRODUCTION}

Multiple input multiple output (MIMO) systems are capable of supporting high-rate, high-integrity transmission [1]. Intensive research efforts have been dedicated to single-user MIMO (SU-MIMO) designs [2], [3]. For multi-user MIMO (MUMIMO) systems, spatial division multiple access (SDMA) has been proposed, where each user's unique channel state information (CSI) is used to distiguish them. Furthermore, in order to simplify the receiver's design at the mobile station (MS) in the context of downlink (DL) transmissions, transmit preprocessing has been proposed to move the part of the required signal processing from the MS to the base station (BS) [4]-[7].

In the simple single-cell scenario only intra-cell interference has been considered [5], [6], but owing to frequency reuse, inter-cell interference is imposed by the other cells [8].

Recently, multiple base station (BS) aided systems have attracted substantial attention [9]-[13]. It has been shown that for a multiple cell system, the achievable system performance can be substantially improved, if cooperative BSs are invoked [9], [10]. For the DL of cooperative BSs, various joint transmission schemes have been proposed. The attainable system capacity was approached by the so-called dirty paper (DP) precoding [9]. However, due to its complexity, linear joint transmission schemes may be more preferred, such as joint zero-forcing (ZF), joint minimum mean square error (MMSE) based DL processing, joint block diagonalization (BD), and joint signal to leakage plus noise ratio (SLNR) based processing [11], [12].

For the cooperative BS aided DL system, each MS may be able to synchronously receive its own signal from different BSs. However, the interference is essentially asychronous and ignoring this asynchronous interference may result in

The financial support of the European Union under the auspices of the Optimix projects, as well as that of the EPSRC UK is gratefully acknowledged. a severe performance loss [11], [12]. Furtunately, the joint $\mathrm{ZF}$ and joint BD based transmission schemes are capable of completely elimitating the interference, which is in contrast to the residual interference experienced after joint MMSE or SLNR processing.

However, the joint ZF based transmission results in several parallel single input single output (SISO) channels, which results in a performance loss compared to the joint detection of the transmitted symbols. Joint BD based transmission is capable of generating several parallel single user multiple input multiple output (SU-MIMO) systems, facilitating the joint DL detection of parallel streams in the transmitted MIMO symbols. However, a specific drawback of the joint BD based transmission is that the resultant SU-MIMO channel will have to be estimated by each MS [14], which may impose an excessive complexity. Recently, singular value decomposition (SVD) based multiuser transmission has been developed in the literature for single-cell systems, which is also capable of decomposing the MU-MIMO system into parallel SUMIMO systems, enabling the joint detection of the transmitted SU-MIMO symbols [15]. Naturally, the effective SU-MIMO channel is entirely determined by each MS's own channel, hence no extra training or overhead is needed compared to BD based transmssion.

In this paper, we extend the SVD-based MU-MIMO transmission from the single-cell case to the multicell scenario. Furthermore, the achievable system performance is evaluated in conjunction with two different power allocation schemes derived for both the BD, SVD and ZF based multicell transmission schemes.

\section{SYSTEM OVERVIEW}

Let us assume that there are $K$ cochannel mobile users arbitrarily distributed in the DL of a multicell system, with $N_{k}$ being the number of receive antennas at each MS and $N_{b}$ the number of adjacent cochannel BSs in the system, with $N_{t}$ being the number of transmit antennas at each BS, respectively as in Fig. 1 for a scenario associated with $N_{b}=$ $K=3$. Assuming non-dispersive or flat-fading conditions, let $H_{j, k}\left(j=1, \cdots, N_{b}, k=1, \cdots, K\right)$ be the small-scale fading channel matrix characterizing the channel between BS $j$ to MS $k$ having zero-mean, unit-variance complex-Gaussian entries, and let $a_{j, k}$ be the corresponding large-scale fading coefficients including the effect of both path-loss and shadow fading. 


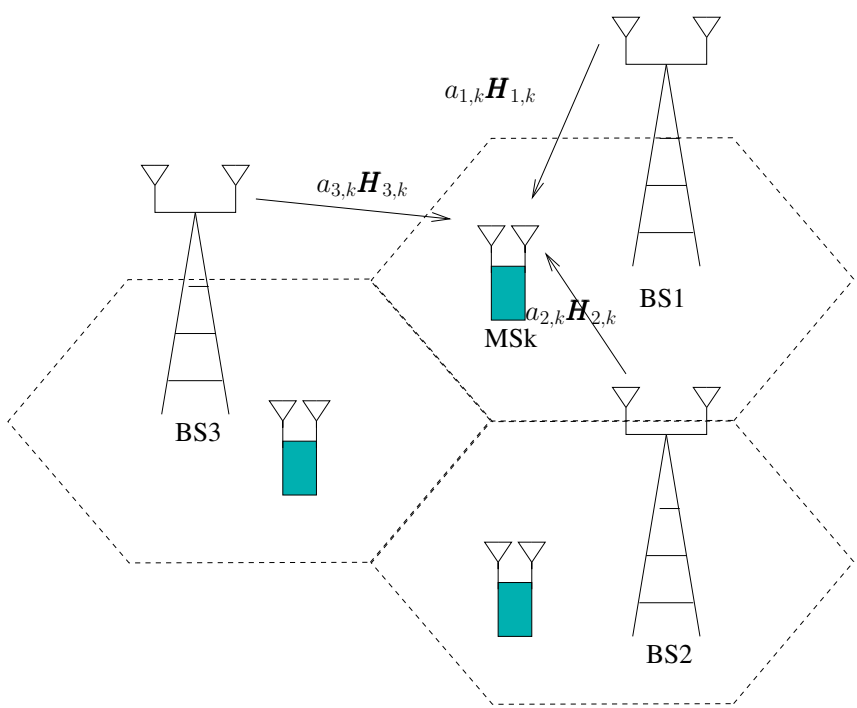

Fig. 1. System overview of multicell cooperation aided transmission.

\section{A. SVD Based Multicell Transmission}

Let us assume that the $N_{k}$-compoment DL transmitted signal vector destined for the $k$ th MS is given by $x_{k}$ and that in the case of BS cooperation, we preprocess $x_{k}$ according to

$$
\boldsymbol{d}_{k}=\boldsymbol{P}_{k} \boldsymbol{\beta}_{k} \boldsymbol{x}_{k},
$$

where $\boldsymbol{P}_{k}$ is an $\left(M \times N_{k}\right)$-element preprocessing matrix with $M=N_{b} \times N_{t}$, which is responsible for cancelling the multiuser interference (MUI), hence resulting in an effective SU-MIMO system. Furthermore, $\boldsymbol{\beta}$ is a $\left(N_{k} \times N_{k}\right)$-element diagonal matrix hosting the power control coefficients.

The signal transmitted from all $N_{b}$ BSs to all $K$ MSs can be expressed as

$$
\boldsymbol{d}=\sum_{k=1}^{K} \boldsymbol{d}_{k}=\sum_{k=1}^{K} \boldsymbol{P}_{k} \boldsymbol{\beta}_{k} \boldsymbol{x}_{k}=\boldsymbol{P} \boldsymbol{\beta} \boldsymbol{x},
$$

where we have

$$
\boldsymbol{P}=\left[\boldsymbol{P}_{1}, \boldsymbol{P}_{2}, \cdots, \boldsymbol{P}_{K}\right],
$$

$\boldsymbol{\beta}=\operatorname{diag}\left[\boldsymbol{\beta}_{1}, \boldsymbol{\beta}_{2}, \cdots, \boldsymbol{\beta}_{K}\right]=\operatorname{diag}\left[\beta_{1}, \beta_{2}, \cdots, \beta_{\left(\sum_{k=1}^{K} N_{k}\right)}\right]$,

and $\boldsymbol{x}$ is a $\left(\sum_{k=1}^{K} N_{k}\right)$-component vector containing the transmitted data, which is given by

$$
\boldsymbol{x}=\left[\boldsymbol{x}_{1}^{T}, \boldsymbol{x}_{2}^{T}, \ldots, \boldsymbol{x}_{K}^{T}\right]^{T} .
$$

Now the signal vector $\boldsymbol{y}_{k}$ received at the $k$ th MS from all $N_{b}$ BSs can be expressed as

$$
\begin{aligned}
\boldsymbol{y}_{k} & =\boldsymbol{H}_{k} \boldsymbol{d}+\boldsymbol{n}_{k} \\
& =\boldsymbol{H}_{k} \boldsymbol{d}_{k}+\boldsymbol{H}_{k} \sum_{j \neq k} \boldsymbol{d}_{j}+\boldsymbol{n}_{k},
\end{aligned}
$$

where the $\left(N_{k} \times M\right)$-element channel matrix $\boldsymbol{H}_{k}$ is given by

$$
\boldsymbol{H}_{k}=\left[a_{1, k} \boldsymbol{H}_{1, k}, a_{2, k} \boldsymbol{H}_{2, k}, \cdots, a_{N_{b}, k} \boldsymbol{H}_{N_{b}, k}\right]
$$

and $\boldsymbol{n}_{k}$ represents the $N_{k}$-element AWGN vector having a zero mean and a covariance matrix of $E\left[\boldsymbol{n}_{k} \boldsymbol{n}_{k}^{H}\right]=\sigma^{2} \boldsymbol{I}_{N_{k}}$,
Furthermore, the all signal vector $\boldsymbol{y}$ received by all the $K$ MSs from all $N_{b}$ Bs can be expressed as

$$
\begin{aligned}
\boldsymbol{y} & =\left[\boldsymbol{y}_{1}, \boldsymbol{y}_{2}, \cdots, \boldsymbol{y}_{K}\right]^{T} \\
& =\left[\boldsymbol{H}_{1}^{T}, \boldsymbol{H}_{2}^{T}, \cdots, \boldsymbol{H}_{K}^{T}\right]^{T} \boldsymbol{d}+\boldsymbol{n} .
\end{aligned}
$$

Let us assume that the rows of $\boldsymbol{H}_{k}(k=1,2, \ldots, K)$ have full rank, i.e. we have $\operatorname{rank}\left(\boldsymbol{H}_{k}\right)=N_{k}$ and that $M \geq$ $\sum_{k=1}^{K} N_{k}$ is satisfied. Then, upon carrying out the SVD of $\boldsymbol{H}_{k}$, we arrive at

$$
\begin{aligned}
\boldsymbol{H}_{k} & =\boldsymbol{U}_{k}\left[\boldsymbol{\Lambda}_{k}^{1 / 2}, \mathbf{0}\right] \boldsymbol{V}_{k}^{H} \\
& =\boldsymbol{U}_{k}\left[\boldsymbol{\Lambda}_{k}^{1 / 2}, \mathbf{0}\right]\left[\begin{array}{c}
\boldsymbol{V}_{k s}^{H} \\
\boldsymbol{V}_{k n}^{H}
\end{array}\right] \\
& =\boldsymbol{U}_{k} \boldsymbol{\Lambda}_{k}^{1 / 2} \boldsymbol{V}_{k s}^{H},
\end{aligned}
$$

where $\boldsymbol{U}_{k}$ and $\boldsymbol{V}_{k}$ are $\left(N_{k} \times N_{k}\right)$-component and $(M \times M)$ component unitary matrices, respectively. Furthermore, $\boldsymbol{\Lambda}_{k}$ is a $\left(N_{k} \times N_{k}\right)$-component diagonal matrix containing the eigenvalues of $\boldsymbol{H}_{k} \boldsymbol{H}_{k}^{H}$, i.e. we have $\boldsymbol{\Lambda}_{k}=$ $\operatorname{diag}\left\{\lambda_{k 1}, \lambda_{k 2}, \cdots, \lambda_{k N_{k}}\right\}$. Finally, $\boldsymbol{V}_{k s}$ in (11) is an $(M \times$ $N_{k}$ )-component matrix, which is constituted by the eigenvectors corresponding to the non-zero eigenvalues of $\boldsymbol{H}_{k}^{H} \boldsymbol{H}_{k}$. By contrast, $\boldsymbol{V}_{k n}$ in (11) is an $\left(M \times\left(M-N_{k}\right)\right)$-component matrix, which is constituted by the eigenvectors corresponding to the zero eigenvalues of $\boldsymbol{H}_{k}^{H} \boldsymbol{H}_{k}$. Similarly, $\boldsymbol{U}_{k}$ consists of the eigenvectors of $\boldsymbol{H}_{k} \boldsymbol{H}_{k}^{H}$.

Upon substituting (11) into (9), we arrive at

$$
\boldsymbol{y}=\boldsymbol{U} \boldsymbol{\Lambda}^{1 / 2} \boldsymbol{V}_{s}^{H} \boldsymbol{P} \boldsymbol{\beta} \boldsymbol{x}+\boldsymbol{n},
$$

where we have

$$
\begin{aligned}
\boldsymbol{U} & =\operatorname{diag}\left[\boldsymbol{U}_{1}, \boldsymbol{U}_{2}, \cdots, \boldsymbol{U}_{K}\right], \\
\boldsymbol{\Lambda} & =\operatorname{diag}\left[\boldsymbol{\Lambda}_{1}, \boldsymbol{\Lambda}_{2}, \ldots, \boldsymbol{\Lambda}_{K}\right], \\
\boldsymbol{V}_{s} & =\left[\boldsymbol{V}_{1 s}, \boldsymbol{V}_{2 s}, \cdots, \boldsymbol{V}_{K s}\right], \\
\boldsymbol{n} & =\left[\boldsymbol{n}_{1}^{T}, \boldsymbol{n}_{2}^{T}, \cdots, \boldsymbol{n}_{K}^{T}\right]^{T} .
\end{aligned}
$$

In (13) $\boldsymbol{U}$ and $\boldsymbol{\Lambda}$ are $\left(\sum_{k=1}^{K} N_{k} \times \sum_{k=1}^{K} N_{k}\right)$-component matrices, $V_{s}$ is a $\left(M \times \sum_{k=1}^{K} N_{k}\right)$-component matrix and $\boldsymbol{n}$ is an AWGN vector, which is Gaussian distributed with zero-mean and a covariance matrix of $\sigma^{2} \boldsymbol{I}_{\sum_{k=1}^{K} N_{k}}$.

For SVD based preprocessing, the preprocessing matrix $\boldsymbol{P}$ can be set to be [15]

$$
\boldsymbol{P}=\left[\boldsymbol{V}_{s}^{H}\right]^{+}=\boldsymbol{V}_{s}\left[\boldsymbol{V}_{s}^{H} \boldsymbol{V}_{s}\right]^{-1}
$$

where we have

$$
\boldsymbol{V}_{s}=\left[\boldsymbol{V}_{1 s}, \boldsymbol{V}_{2 s}, \cdots, \boldsymbol{V}_{K s}\right]
$$

and $\left[\boldsymbol{V}_{s}^{H}\right]^{+}$denotes the pseudo-inverse of the matrix $\boldsymbol{V}_{s}^{H}$. Consequently, the resultant signal received at the $k$ th $\mathrm{MS}$ is given by

$$
\boldsymbol{y}_{k}=\boldsymbol{U}_{k} \boldsymbol{\Lambda}_{k}^{1 / 2} \boldsymbol{\beta}_{k} \boldsymbol{x}_{k}+\boldsymbol{n}_{k}, k=1,2, \ldots, K .
$$




\section{The Achievable Maximum Transmission Rate}

The major difference between multi-cell and single-cell transmission is that the power constraints have to be considered on a per-BS basis, i.e. the average transmit power of the $j$ th $\mathrm{BS}$ is limited by $P_{j}$.

For multicell transmission, the preprocessing matrix for the $k$ th MS can be expressed as

$$
\boldsymbol{P}_{k}=\left[\boldsymbol{P}_{k, 1}^{T}, \boldsymbol{P}_{k, 2}^{T}, \cdots, \boldsymbol{P}_{k, N_{b}}^{T}\right],
$$

where the $\left(N_{t} \times N_{k}\right)$-dimensional matrix $\boldsymbol{P}_{k, j}$ is the preprocessing matrix configured for transmission from the $j$ th BS to the $k$ th MS.

Furthermore, let the $\left(N_{t} \times \sum_{k=1}^{K} N_{k}\right)$-dimensional matrix $\overline{\boldsymbol{P}}^{j}$ denote the preprocessing matrix configured for transmission from the $j$ th $\mathrm{BS}$ to all the $K \mathrm{MSs}$, which is given by

$$
\overline{\boldsymbol{P}}_{j}=\left[\boldsymbol{P}_{1, j}, \boldsymbol{P}_{2, j}, \cdots, \boldsymbol{P}_{K, j}\right],
$$

which represents the preprocessing matrix configured for transmission from the $j$ th $\mathrm{BS}$ to all the $K$ MSs.

In order to meet the per-BS power constraints, we have to satisfy

$$
\begin{aligned}
\operatorname{Trace}\left[\overline{\boldsymbol{P}}_{j} \boldsymbol{\beta} \boldsymbol{x} \boldsymbol{x}^{H} \boldsymbol{\beta}^{H} \overline{\boldsymbol{P}}_{j}^{H}\right] & =\operatorname{Trace}\left[\boldsymbol{\beta} \boldsymbol{\beta}^{H} \overline{\boldsymbol{P}}_{j}^{H} \overline{\boldsymbol{P}}_{j}\right] \\
& =\sum_{i=1}^{N_{k}} \beta_{i}^{2}\left[\overline{\boldsymbol{P}}_{j}^{H} \overline{\boldsymbol{P}}_{j}\right]_{i, i} \leq P_{j},
\end{aligned}
$$

where $\left[\overline{\boldsymbol{P}}_{j}^{H} \overline{\boldsymbol{P}}_{j}\right]_{i, i}$ is the $i$ th diagonal element of the matrix $\left[\overline{\boldsymbol{P}}_{j}^{H} \overline{\boldsymbol{P}}_{j}\right]$ and Trace $(\cdot)$ denotes the trace of the argument.

The maximum achievable average transmission rate per user per antenna is given by

$$
\begin{aligned}
R_{S V D} & =\max \frac{1}{\sum_{k=1}^{K} N_{k}} \sum_{i=1}^{K} \log _{2}\left|\boldsymbol{I}_{N_{r}}+\frac{1}{\sigma^{2}} \boldsymbol{U}_{k} \boldsymbol{\Lambda}_{k}^{1 / 2} \boldsymbol{\beta}_{k}^{2} \boldsymbol{\Lambda}_{k}^{1 / 2} \boldsymbol{U}_{k}^{H}\right| \\
& =\max \frac{1}{\sum_{k=1}^{K} N_{k}} \sum_{i=1}^{K} \log _{2}\left|\boldsymbol{I}_{N_{r}}+\frac{1}{\sigma^{2}} \boldsymbol{\Lambda}_{k}^{1 / 2} \boldsymbol{\beta}_{k}^{2} \boldsymbol{\Lambda}_{k}^{1 / 2}\right| \\
& =\max \frac{1}{\sum_{k=1}^{K} N_{k}} \sum_{i=1}^{K} \log _{2}\left|\boldsymbol{I}_{N_{r}}+\frac{1}{\sigma^{2}} \boldsymbol{\beta}_{k}^{2} \boldsymbol{\Lambda}_{k}\right| \\
& =\max \frac{1}{\sum_{k=1}^{K} N_{k}} \sum_{i=1}^{K} \sum_{l=1}^{N_{k}} \log _{2}\left(1+\frac{1}{\sigma^{2}} \lambda_{k} \beta_{k j}^{2}\right)
\end{aligned}
$$

under the constraints of (19).

The above optimization problem may be solved for example, by the interior-point method as recommended in [13]. However, since it can be complex to deal with, we resort to a less complex suboptimal solution in this paper.

\section{A. Scaled Suboptimal Power Allocation}

First, the so-called scaled power allocation is considered [13]. In this case, the power allocation is performed firstly by assuming that all BSs can jointly pool their power, i.e. the maximum achievable average rate is obtained by

$$
R_{J o i n t}=\frac{1}{\sum_{k=1}^{K} N_{k}} \sum_{i=1}^{K} \sum_{l=1}^{N_{k}} \log _{2}\left(1+\frac{1}{\sigma^{2}} \lambda_{k} \beta_{k j}^{2}\right)
$$

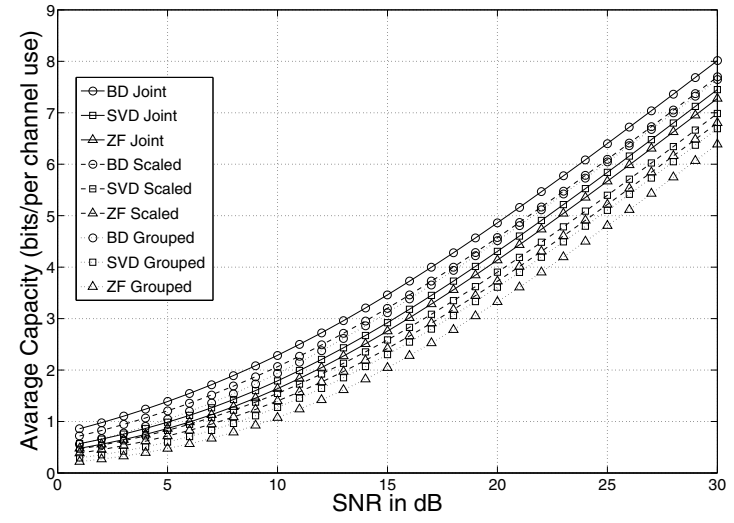

Fig. 2. Average Capacity versus average SNR performance for BD, SVD and ZF based Multicell Transmission for the case of three BSs and three MSs.

under the constraint of

$$
\operatorname{Trace}\left[\boldsymbol{\beta} \boldsymbol{\beta}^{H} \boldsymbol{P}^{H} \boldsymbol{P}\right]=\sum_{i=1}^{\sum_{i} N_{k}} \beta_{i}^{2}\left[\overline{\boldsymbol{P}}^{H} \overline{\boldsymbol{P}}\right]_{i, i} \leq \sum_{j=1}^{N_{b}} P_{j}
$$

Then, the power allocation matrix is scaled by a factor of $\mu$, which is given by

$$
\mu=\min _{j=1,2, \cdots, N_{b}} \frac{P_{j}}{\sum_{i=1}^{\sum_{k} k} \beta_{i}^{2}\left[\overline{\boldsymbol{P}}_{j}^{H} \overline{\boldsymbol{P}}_{j}\right]_{i, i}} .
$$

Therefore, the maximum achievable average rate of this scheme is obtained by

$$
R_{\text {Scaled }}=\frac{1}{\sum_{k=1}^{K} N_{k}} \sum_{i=1}^{K} \sum_{l=1}^{N_{k}} \log _{2}\left(1+\mu \frac{1}{\sigma^{2}} \lambda_{k} \beta_{k j}^{2}\right)
$$

\section{B. Grouped Suboptimal Power Allocation}

Another suboptimal power allocation policy is to divide the transmitted symbol vector into $N_{b}$ groups, where each symbol of the same group is assigned the same power coefficient $u_{j}$, and the power vector $\boldsymbol{u}=\left[u_{1}, \cdots, u_{N_{b}}\right]^{T}$ assigned tp all the MSs can be expressed as [11].

$$
\boldsymbol{u}=\boldsymbol{Q}^{-1} \boldsymbol{P},
$$

where $Q$ is an $\left(N_{b} \times N_{b}\right)$-element matrix given by

$$
\boldsymbol{Q}=\left[\begin{array}{ccc}
\operatorname{Tr} P_{(1,1)}^{H} P_{(1,1)} & \cdots & \operatorname{Tr} P_{\left(1, N_{b}\right)}^{H} P_{\left(1, N_{b}\right)} \\
\operatorname{Tr} P_{(2,1)}^{H} P_{(2,1)} & \cdots & \operatorname{Tr} P_{\left(2, N_{b}\right)}^{H} P_{\left(2, N_{b}\right)} \\
\vdots & \ddots & \vdots \\
\operatorname{Tr} P_{\left(N_{b}, 1\right)}^{H} P_{\left(N_{b}, 1\right)} & \cdots & \operatorname{Tr} P_{\left(N_{b}, N_{b}\right)}^{H} P_{\left(\left(N_{b}, N_{b}\right)\right.}
\end{array}\right]
$$

where $\boldsymbol{P}_{(i, j)}$ is the precoding matrix configured for DL transmission at $i$ th BS to the $j$ th group of transmitted data. In the scenario where no feasible solution exists, all the symbols are assigned the same power $\beta$, which is given by [11]

$$
\beta^{2}=\min _{j=1,2, \cdots, N_{b}} \frac{P_{j}}{\sum_{i=1}^{\sum_{k} N_{k}\left[\overline{\boldsymbol{P}}_{j}^{H} \overline{\boldsymbol{P}}_{j}\right]_{i, i}} .}
$$




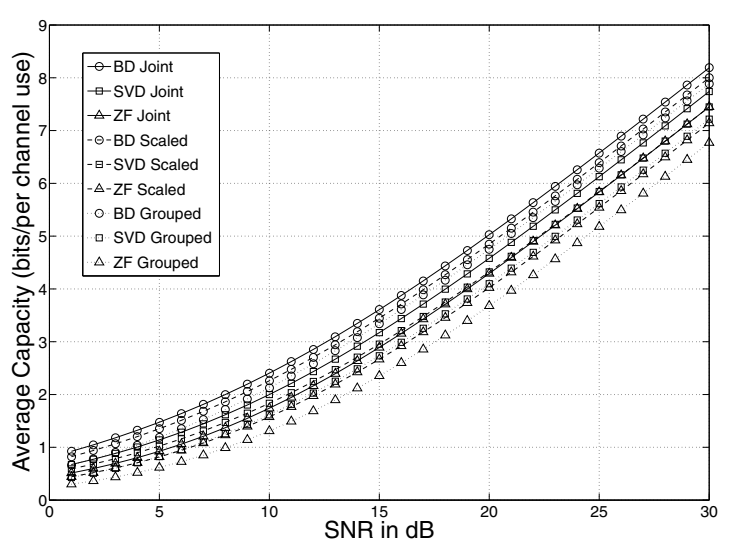

Fig. 3. Average Capacity versus average SNR performance for BD, SVD and ZF based Multicell Transmission for the case of two BSs and two MSs.

\section{Simulation Results}

In this section, simulation results are presented to characterize the achievable performance of SVD-based multicell transmission in conjunction with the different power allocation schemes considered. The results are also compared to those of $\mathrm{BD}$ and $\mathrm{ZF}$ based multicell transmission schemes. Specifically,the large-scale fading is assumed to be $a_{j, k}^{2}=1$ for $j=k$, otherwise $a_{j, k}^{2}=0.5$ as in [11], which ignores the shadow fading. Furthermore, the transmission power of each BS is assumed to be the same, given by $P_{t}$ and the SNR is defined as $P_{t} / \sigma^{2}$.

In Fig.2, the maximum achievable average capacity of BD, SVD and ZF based multicell transmission is investigated, where we assume that there are three BSs and three MSs. Each of the BSs supports a single MS within its coverage area. Furthermore, the number of BS transmit antennas is assumed to be $N_{t}=2$ and the number of receive antennas at the MS is $N_{k}=2$. Furthermore, the performance of joint power allocation scheme of (21) is also plotted as an upper bound. As we can see from Fig.2, the scaled suboptimal power allocation scheme of (24) outperforms the grouped power allocation scheme of (25) for any of the transmission strategies considered. Furthermore, the BD based scheme achieves the best performance, since it has a unitary preprocessing matrix [11], which does not reduce the effective power of each symbol. However, in order to generate the resultant SU-MIMO system CSI for each MS, extra trainning or overhead is invoked for the BD based scheme [14]. Moveover, the SVD based scheme has an approximately $1 \mathrm{~dB}$ to $1.5 \mathrm{~dB}$ performance gain over the ZF based scheme, since SVD based scheme results in joint reception of the transmitted SU-MIMO streams, while the ZF based scheme results in parallel SISO channels. Similar trends can also be observed in Fig.3, where we assume that there are two BSs and two MSs, where each of the BSs has a single MS within its coverage area. Furthermore, the number of BS transmit antennas is assumed to be $N_{t}=2$ and the number of recieve antennas at the MS is $N_{k}=2$.

\section{CONCLUSIONS}

In this paper, we investigated the extension of SVD based multiuser transmission from the single cell case to the multicell scenario, where the multiple BSs may cooperate during their transmissions. We also compared the SVD based BS cooperation to the corresponding $\mathrm{BD}$ and $\mathrm{ZF}$ based schemes, while using different power allocation policies. The BD based scheme is capable of achieving the best performance, while the SVD based scheme is suboptimal. However, the BD based scheme requires extra training for generating the effective SU-MIMO CSI, while the SVD based scheme avoids this requirement. Hence, the SVD based scheme is less complex than the BD based one.

\section{REFERENCES}

[1] I. E. Telatar, "Capacity of multi-antenna Gaussian channels," European Transactions on Telecommunications, vol. 10, pp. 585-595, May 1999.

[2] P. W. Wolniansky, G. J. Foschini, G. D. Golden, and R. A. Valenzuela, "V-BLAST: an architecture for realizing very high data rates over the rich-scattering wireless channel," in International Symposium on Signals, Systems, and Electronics, (Pisa, Italy), pp. 295-300, 29 September - 2 October 1998.

[3] V. Tarokh, N. Seshadri, and A. R. Calderbank, "Space-time codes for high data rate wireless communication: performance criterion and code construction," IEEE Transactions on Information Theory, vol. 44 pp. 744-765, March 1998.

[4] M. Joham, W. Utschick, and J. A. Nossek, "Linear transmit processing in MIMO communications systems," IEEE Transactions on Signal Processing, vol. 53, pp. 2700- 2712, August 2005.

[5] Q. H. Spencer, A. L. Swindlehurst, and M. Haardt, "Zero-forcing methods for downlink spatial multiplexing in multiuser MIMO channels," IEEE Transactions on Signal Processing, vol. 52, pp. 461 - 471, February 2004.

[6] L. Choi and R. D. Murch, "A transmit preprocessing technique for multiuser MIMO systems using a decomposition approach," IEEE Transactions on Wireless Communications, vol. 3, pp. 20- 24, January 2004.

[7] M. Morelli and L. Sanguinetti, "A novel prefiltering technique for downlink transmissions in TDD MC-CDMA systems," IEEE Transactions on Wireless Communications, vol. 4, pp. 2064 - 2069, September 2005.

[8] S. Shim, J. S. Kwak, R. W. H. Jr., and J. G. Andrews, "Block diagonalization for multi-user mimo with other-cell interference," IEEE Transactions on Wireless Communications, vol. 7, pp. 2671-2681, July 2008.

[9] S. Shamai and B. M. Zaidel, "Enhancing the cellular downlink capacity via co-processing at thetransmitting end," in IEEE VTS 53rd Vehicular Technology Conference Spring, (Rhodes, Greece), pp. 1745 - 1749, 6 9 May 2001.

[10] M. K. Karakayali, G. J. Foschini, R. A. Valenzuela, and R. D. Yates, "On the maximum common rate achievable in a coordinated network," in IEEE International Conference on Communications, (Istanbul, Turky), pp. 4333 - 4338, 11 - 15 June 2006.

[11] H. Zhang and H. Dai, "Cochannel interference mitigation and cooperative processing in downlink multicell multiuser MIMO networks," EURASIP Journal on Wireless Communications and Networking, vol. 2004, pp. 222 - 235, 4th Quarter 2004.

[12] H. Zhang, N. B. Mehta, A. F. Molisch, J. Zhang, and H. Dai, "Asynchronous interference mitigation in cooperative base station systems," IEEE Transactions on Wireless Communications, vol. 7, pp. 155 - 165, January 2008.

[13] J. Zhang, C. R, J. G. Andrews, and R. W. H. Jr., "Coordinatedm multicell MIMO systems with cellular block diagonalization," in Conference Record of the Forty-First Asilomar Conference on Signals, Systems and Computers, (Pacific Grove, California, United States), pp. 1669 - 1673, 4-7 November 2007.

[14] S. Shim, C. B. Chae, and R. W. Heath, "A lattice-based MIMO broadcast precoder with block diagonalization for multi-stream transmission," in IEEE Global Telecommunications Conference, (San Francisco, CA, USA), pp. 1 - 5, 27 November - 1 December 2006. 
[15] W. Liu, L. L. Yang, and L. Hanzo, "SVD assisted multiuser transmitter and multiuser detector design for MIMO systems," accepted by IEEE Transactions on Vehicular Technology. 